\title{
On the asymptotic stability of solutions of functional differential equations
}

\author{
by J. TERJÉkI (Szeged)
}

\begin{abstract}
The paper deals with stability properties of the zero solution of functional differential equations of retarded type with infinite delay. The main result is a refinement of the method, used by Mikolajska [7], adopted here to suit the investigation of asymptotic stability.
\end{abstract}

\section{Introduction. Terminology}

Denote by $R, R^{+}, R^{-}$the real, non-negative, non-positive real numbers resp. and by $R^{n}$ the set of all ordered $n$-tuples. Let $|\cdot|$ be a norm in $R^{n}$. $C$ denotes the Banach space of bounded continuous functions $\varphi: R^{-} \rightarrow R^{n}$ with the norm $\|\varphi\|=\sup \left\{|\varphi(s)|: s \in R^{-}\right\}$. Given a $t \in R^{+}$and a function $x:(-\infty, t] \rightarrow R^{n}$, which is bounded and continuous, we define $x_{t} \in C$ by $x_{l}(s)=x(t+s)$ for $s \in R^{-}$. For a given $\varrho$ with $0<\varrho \leqslant \infty$ write $C_{0}$ $=\{\varphi \in C:\|\varphi\|<\varrho\} . \bar{C}_{\varrho}$ is the closure of $C_{\varrho}$.

Suppose there is given a functional $F(t, \varphi)$.

Consider the functional differential equation

$$
\dot{x}(t)=F\left(t, x_{t}\right) \text {. }
$$

In the followings we always assume:

$1^{0}$ For every $\left(t_{0}, \varphi_{0}\right) \in R^{+} \times C_{e}$ there exist a number $\varepsilon>0$ and a continuous function $x:\left(-\infty, t_{0}+\varepsilon\right) \rightarrow R^{n}$ (called the solution of (1.1) through $\left.\left(t_{0}, \varphi_{0}\right)\right)$ such that $x_{t_{0}}=\varphi_{0}, x$ is derivable and absolutely continuous on $\left(t_{0}, t_{0}+\varepsilon\right)$, and satisfies (1.1) in this interval (see Halanay and Yorke [2]).

$2^{\circ}$ If the solution $x$ is non-continuable on $(-\infty, T)$ with $T<\infty$, then $\limsup |x(t)|=\varrho$.

$$
3^{\circ} F^{\prime}(t, 0)=0 \text { for } t \in R^{+} \text {. }
$$

We shall denote by $x\left(t_{0}, \varphi_{0}\right)$ any non-continuable solution of (1.1) through $\left(t_{0}, \varphi_{0}\right)$, by $x\left(t ; t_{0}, \varphi_{0}\right)$ its value at $t$ and by $x_{t}\left(t_{0}, \varphi_{0}\right)$ the corresponding element of $C$. 
Now, to avoid ambiguities, we list the stability definitions used in what follows.

We shall say that the zero solution of (1.1) is

(i) stable at $t_{0}$, whenever for each $\varepsilon>0$ there exists $\delta\left(\varepsilon, t_{0}\right)>0$, such that for any $\varphi_{0} \in \bar{C}_{\delta\left(\varepsilon, t_{0}\right)}$, each solution $x\left(t_{0}, \varphi_{0}\right)$ is defined on $R$ and satisfies

$$
\left|x\left(t ; t_{0}, \varphi_{0}\right)\right| \leqslant \varepsilon, \quad t \geqslant t_{0} ;
$$

(ii) stable, if for every $t_{0} \in R^{+}$it is stable at $t_{0}$;

(iii) uniformly stable, whenever for every $\varepsilon>0$ there is a $\delta(\varepsilon)>0$ such that if $\varphi_{0} \in \bar{C}_{\delta(\theta)}, t_{0} \in R^{+}$, then any solution $x\left(t_{0}, \varphi_{0}\right)$ is defined on $R$ and satisfies (1.2);

(iv) equi-asymptotically stable, if it is stable and for every $t_{0} \in R^{+}$ there exists $\Delta\left(t_{0}\right)>0$ such that, for arbitrary $\varphi_{0} \in \vec{C}_{\Delta\left(t_{0}\right)}$, any $x\left(t_{0}, \varphi_{0}\right)$ is defined on $R$, and for'every $\eta>0$ there is $T\left(t_{0}, \eta\right)>0$ such that

$$
\left|x\left(t ; t_{0}, \varphi_{0}\right)\right| \leqslant \eta, \quad t \geqslant t_{0}+T\left(t_{0}, \eta\right) ;
$$

(v) strictly equi-asymptotically stable, if it is uniformly stable and there exists $\Delta>0$ such that any $x\left(t_{0}, \varphi_{0}\right)$ is deifned on $R$ for $t_{0} \in R^{+}$and $\varphi_{0} \in \bar{C}_{\Delta}$, and for arbitrary $\eta>0$ there is a $T\left(t_{0}, \eta\right)>0$ such that (1.3) holds;

(vi) uniformly asymptotically stable, if it is strictly equi-asymptotically stable and for every $\eta>0$ there exists $T(\eta)>0$ such that if $t_{0} \in R^{+}$, $\varphi_{0} \in \bar{C}_{\Delta}$, then

$$
\left|x\left(t ; t_{0}, \varphi_{0}\right)\right| \leqslant \eta, \quad t \geqslant t_{0}+T(\eta) ;
$$

(vii) exponentially stable, if there exist constants $\Delta>0, B \geqslant 1, a>0$ such that for $t_{0} \in R^{+}, \varphi_{0} \in \bar{C}_{\Delta}$, any $x\left(t_{0}, \varphi_{0}\right)$ is defined on $R$ and the following inequality holds:

$$
\left|x\left(t ; t_{0}, \varphi_{0}\right)\right| \leqslant B\left\|\varphi_{0}\right\| e^{-a\left(t-t_{0}\right)}, \quad t \geqslant t_{0} .
$$

We denote by $K$ the set of strictly increasing, vanishing at zero continuous functions mapping $R^{+}$into $R^{+}$.

In what follows we assume the existence of functions $V, \omega, \omega_{1}, \omega_{2}, \ldots$ : $R \times R^{n} \rightarrow R$, which are supposed to be continuous everywhere and continuously partially differentiable on $R \times\left(R^{n}-\{0\}\right)$ with respect to each of their variables. The function $V$ is positive definite, i.e. there exists a function $a \in K$ such that $a(|x|) \leqslant V(t, x)$ holds for $t \in R^{+}$and $x \in R^{n}$.

For every $V$ (similarly for $\omega, \omega_{1}, \omega_{2}, \ldots$ ) we define the function $\dot{V}: R^{+} \times C_{\mathrm{e}} \rightarrow R$ by

$$
\dot{V}(t, \varphi)=\frac{\partial V}{\partial t}(t, \varphi(0))+\sum_{i=1}^{n} \frac{\partial V}{\partial x_{i}}(t, \varphi(0)) F_{i}(t, \varphi),
$$


which is called the total derivative of $\nabla$ by virtue of equation $(1.1)\left(F_{i}(t, \varphi)\right.$ is the $i$-th component of $F(t, \varphi)$ ).

Obviously, for every solution $x\left(t_{0}, \varphi_{0}\right)$ of $(1.1)$, if $x\left(t ; t_{0}, \varphi_{0}\right) \neq 0$ for $t \in\left[t_{0}, T\right]\left(T>t_{0}\right)$, then $\nabla\left(t, x\left(t ; t_{0}, \varphi_{0}\right)\right)$ is absolutely continuous on $\left[t_{0}, T\right]$ and

$$
\frac{d}{d t} V\left(t, x\left(t ; t_{0}, \varphi_{0}\right)\right)=\dot{V}\left(t, x_{t}\left(t_{0}, \varphi_{0}\right)\right), \quad t \in\left(t_{0}, T\right) .
$$

Several important special cases of (1.1) have been investigated. One of them is the class of equations treated by Krasovskii [4], where $F$ has poperty: there exists $h \geqslant 0$ such that, if $\varphi, \psi \in C$ and $\varphi(s)=\psi(s)$ for $s \in[-h, 0]$, then the equality $F(t, \varphi)=F(t, \psi)$ holds for all $t \in R^{+}$. In this case equation (1.1) is called an equation with finite delay.

The stability behaviour of these equations - as it was proved by Krasovskii - can be studied in a way analogous to Lyapunov's method [5], with Lyapunov functions replaced by Lyapunov functionals. With the aid of these functionals one can state necessary and sufficient conditions for some stability properties and can answer some special problems (e.g., that of stability in the first approximation). But in concrete problems the construction of these functionals - without using the explicit form of the solutions - is rather complicated. Therefore some other methods, with ensure only sufficiency, but can be applied easily, are of great importance. Such a result is Razumikhin's method [8], where the stability is ensured with the use of Lyapunov functions. Razumikhin proved the following theorem on equations with finite delay. If there exists a function $\nabla$ with

$$
\dot{V}(t, \varphi) \leqslant 0
$$

on the set

$$
\left\{(t, \varphi): V(t+s, \varphi(s)) \leqslant V(t, \varphi(0)), s \in R^{-}\right\},
$$

then the zero solution of (1.1) is stable. Razumikhin tried to apply his method to the investigation of asymptotic stability, but his result - as it was observed by Mikołajska [6] - was incorrect. A fairly well applicable theorem on asymptotical stability was obtained by Krasovskii [4], who proved (for equations with finite delay) that if instead of (1.4) we assume the inequality

$$
\dot{V}(t, \varphi) \leqslant-c(|\varphi(0)|)
$$

on the set

and if

$$
\left\{(t, \varphi): V(t+s, \varphi(s))<f(V(t, \varphi(0))), s \in R^{-}\right\}
$$

$$
V(t, x) \leqslant b(|x|), \quad t \in R^{+}, x \in R^{n},
$$


where $c, f, b \in K$ and $f(v)>v$ for $v>0$, then the zero solution of (1.1) is asymptotioally stable. Later on, assuming the same conditions Driver [1] proved the uniformly asymptotical stability of the zero solution.

In 1969 Mikołajska [7] obtained a result more general than that of Razumikhin's. She studied - using functions $\omega_{1}, \omega_{2}, \ldots, \omega_{n}$ - the stability at 0 of the zero solution of some special equations with finite delay. In the scalar case there are assumed (instead of the positive definiteness of the function $\omega$ ) the inequality $x \omega(t, x)>0$ for $x \neq 0$ and the positive definiteness of $|\omega(t, x)|$. Inequality (1.4) was used in a sharper form $\varphi(0) \dot{\omega}(t, \varphi)<0$, required, however, to hold only on the set

$$
\left\{(t, \varphi): \alpha|\omega(t, \varphi(0))| \leqslant|\omega(t+s, \varphi(s))| \leqslant \mid \omega\left(t, \varphi(0)||, s \in R^{-}\right\} .\right.
$$

Seifer [8] studied the stability at 0 of the zero solution of equations with infinite delay of the type in which $F(t, \varphi)=F(t, \psi)$ if $\varphi(s)=\psi(s)$ for $s \in[-t, 0]$. It was shown that if there exists a function $V$ with (1.4) on the set (1.7) and with (1.8), then the zero solution is stable at 0 . He gives an example shoving that Krasovskii's theorem mentioned above is not valid for equations with infinite delay.

Our main result is a refinement of the method used by Mikołajska, applied so as to suit the investigation of asymptotical stability. As corollaries we get an extension of the theorems of Razumikhin, Krasovskii, Mikołajska, regarding (1.1), and a generalization of Seifert's result.

We also give examples to show that our results can be used in several cases in which the above cited theorems cannot be applied.

\section{The exposition of the method}

In this section we present our method giving some relatively simple but not too special - results. We use a single function $V$. The results will be formulated in Section 3 with more than one auxiliary function.

Besides the function $V$ (with the poperty: $a(|x|) \leqslant V(t, x), t \in R^{+}$, $x \in R^{n}$, where $\left.a \in K\right)$, let there be given $\alpha, \beta \in K$ with $a(r)<\beta(r) \leqslant r$ for $r>0$ and $f: R^{-} \times R^{+} \rightarrow R^{+}$such that $f(0, v) \leqslant v$ for $v \in R^{+}$and $f(s, v)$ is increasing in $s$ for fixed $v$. For a given $r>0, t \in R^{+}$and $\bar{t} \in R^{+}$with $\bar{t} \leqslant t$, we define the set

$$
\begin{aligned}
Z(t, \bar{t}, r)=\left\{\varphi \in C_{\mathrm{e}}: \alpha(r) \leqslant \nabla(t+s, \varphi(s)) \leqslant r, s \in[\bar{t}-t, 0]\right. & \\
& \left.f(s, V(t+s, \varphi(s))) \leqslant r, s \in R^{-}\right\}
\end{aligned}
$$

and introduce the notation:

$$
z(t, \bar{t}, r)=\sup \{\dot{V}(t, \varphi): \varphi \in Z(t, \bar{t}, r)\}
$$


Moreover, let a function $h: R^{+} \rightarrow R^{+}$be given, $h(t) \leqslant t$, and let us define the set

$$
N(t)=\bigcup_{r>0}\left(Z(t, h(t), r) \cap\left\{\varphi \in C_{\mathrm{a}}: \nabla(t, \varphi(0)\}=\beta(r)\right\}\right) .
$$

THEOREM 1. Assume that we can find functions $V, a, \beta, f, h$ with the above properties. Then:

$1^{\circ}$ The conditions

(A) for every $t_{0} \in R^{+}$there is $b_{t_{0}} \in K$ with $\nabla\left(t_{0}+s, \varphi(s)\right) \leqslant b_{t_{0}}(\|\varphi\|)$, $s \in R^{-}, \varphi \in C_{e}$,

(B) $\dot{V}(t, \varphi) \leqslant 0$ if $t \in R^{+}, h(t)>0$ and $\varphi \in N(t)$,

(C) for every $t \in R^{+}, \quad r \in(0, a(\varrho)), \tau \in(h(t), t]$ and $s \in[\tau, t]$ the set $Z(s, \tau, r)$ is not empty, the function $z(s, \tau, r)$ is integrable with respect to $s$ and

$$
\int_{\tau}^{t} z(s, \tau, r) d s<\beta(r)-\alpha(r),
$$

imply that the zero solution of (1.1) is stable.

$2^{\circ}$ If (A), (B), (C) hold together with

(D) $\lim (\sup \{f(s, v): 0 \leqslant v \leqslant a(\varrho)\})=0$,

(E) $\beta(r)<r$ for every $0<r$,

(F) for every $r \in(0, a(\varrho)), t_{0} \in R^{+}$there exists an interval $\left[T_{1}\left(t_{0}, r\right)\right.$, $\left.T_{2}\left(t_{0}, r\right)\right] \subset\left(t_{0}, \infty\right)$ such that $Z\left(s, t_{0}, r\right)$ is not empty for $s \in\left[T_{1}\left(t_{0}, r\right)\right.$, $\left.T_{2}\left(t_{0}, r\right)\right]$, the function $z\left(s, t_{0}, r\right)$ is integrable with respect to $s$ and

$$
\int_{T_{1}\left(t_{0}, r\right)}^{T_{2}\left(t_{0}, r\right)} z\left(s, t_{0}, r\right) d s \leqslant \alpha(r)-r,
$$

then the zero solution of (1.1) is equi-asymptotically stable.

First we prove some lemmas:

LEMIMA 1. Let $v(t)$ be continuous on $\left[t_{1}, t_{2}\right]$ differentiable on $\left(t_{1}, t_{2}\right)$ and let $v\left(t_{1}\right)<v\left(t_{2}\right)$; then there exists $t^{*} \in\left(t_{1}, t_{2}\right)$ such that $v(s)<v\left(t^{*}\right)$ if $s \in\left[t_{1}, t^{*}\right)$ and $\dot{v}\left(t^{*}\right)>0$.

The proof goes easily by using e.g. the function

$$
f(t)=\frac{1}{3} v\left(t_{2}\right)+\frac{2}{3} v\left(t_{1}\right)+\frac{1}{3} \frac{v\left(t_{2}\right)-v\left(t_{1}\right)}{t_{2}-t_{1}}\left(t-t_{1}\right) .
$$

Let $x\left(t_{0}, \varphi_{0}\right)$ be a solution of (1.1) defined on $(-\infty, T)$, where $T>t_{0}$. Put $v(t)=V\left(t, x\left(t ; t_{0}, \varphi_{0}\right)\right)$.

LEMnA 2. Suppose that conditions (B) and (C) are satisfied. For arbitrarily given $r$ and $t_{1}\left(0<r<a(\varrho), t_{0} \leqslant t_{1}<T\right)$, if $f\left(s, v\left(t_{1}+s\right)\right) \leqslant r$, $s \in R^{-}$and $v\left(t_{1}\right) \leqslant " \alpha(r)$, then $v(t) \leqslant \beta(r), t \in\left[t_{1}, T\right)$. 
Proof. Suppose the assertion to be false, i.e., assume that the set $Q=\left\{t: v(t)>\beta(r), t \in\left[t_{1}, T\right)\right\}$ is non-empty. Put $t_{3}=\inf Q$. Then $v\left(t_{3}\right)$ $=\beta(r)$. Choose arbitrarily $t_{5} \in Q$. By Lemma 1 there exists $t_{4} \in\left(t_{3}, t_{5}\right)$ with $\dot{v}\left(t_{4}\right)>0$ and $v(t)<v\left(t_{4}\right)$ for $t \in\left[t_{3}, t_{4}\right)$. Let $r^{\prime}=\beta^{-1}\left(v\left(t_{4}\right)\right)$. According to the definition of $t_{3}, v\left(t_{1}\right) \leqslant \alpha(r)<\alpha\left(r^{\prime}\right)$ and $v(t) \leqslant \beta(r)<\beta\left(r^{\prime}\right)$ for $t \in\left[t_{1}, t_{3}\right]$. Therefore there is $t_{2} \in\left(t_{1}, t_{4}\right)$ such that $v\left(t_{2}\right)=\alpha\left(r^{\prime}\right)$ and

$$
\alpha\left(r^{\prime}\right)<v(t)<\beta\left(r^{\prime}\right), \quad t \in\left(t_{2}, t_{4}\right) .
$$

Now we show that for any $t \in\left[t_{1}, t_{4}\right]$

$$
f(s, v(t+s))<r^{\prime}, \quad s \in R^{-} .
$$

Indeed, if $s \in\left(t_{1}-t, 0\right)$, then $v(t+s)<\beta\left(r^{\prime}\right)$ and thus $f(s, v(t+s))$ $\leqslant v(t+s)<\beta\left(r^{\prime}\right) \leqslant r^{\prime}$. If $s \in\left(-\infty, t_{1}-t\right]$, then according to the monotonicity of $f$ and the assumptions of Lemma 2 we have

$$
\begin{aligned}
f(s, v(t+s)) & =f\left(\left(s+t-t_{1}\right)+t_{1}-t, v(t+s)\right) \\
& \leqslant f\left(s+t-t_{1}, v\left(\left(s+t-t_{1}\right)+t_{1}\right)\right) \leqslant r<r^{\prime} .
\end{aligned}
$$

There are two possibilities:

(a) $t_{2} \leqslant h\left(t_{4}\right)$

(b) $t_{2}>h\left(t_{4}\right)$.

Case (a) leads to a contradiction because, according to (2.1), (2.2), $t_{2}>0$ and the definition of $r^{\prime}$, we have $x_{t_{4}} \in N\left(t_{4}\right), h\left(t_{4}\right)>0$, and therefore, on account of condition (B), $\dot{v}\left(t_{4}\right)=\dot{V}\left(t_{4}, x_{t_{4}}\left(t_{0}, \varphi_{0}\right)\right) \leqslant 0$ (contrary to the choice of $t_{4}$ ).

If (b) is satisfied, it follows from (c) that $\int_{t_{2}}^{t_{4}} z\left(s, t_{2}, r^{\prime}\right) d s<\beta\left(r^{\prime}\right)-\alpha\left(r^{\prime}\right)$. But from (2.1) and (2.2) we have $x_{s}\left(t_{0}, \varphi_{0}\right) \in Z\left(s, t_{2}, r^{\prime}\right), s \in\left[t_{2}, t_{4}\right]$; therefore $\dot{v}(s) \leqslant z\left(s, t_{2}, r^{\prime}\right)$ and so we have the contradiction:

$$
\begin{aligned}
\beta\left(r^{\prime}\right)-\alpha\left(r^{\prime}\right) & =v\left(t_{4}\right)-v\left(t_{2}\right)=\int_{t_{2}}^{t_{4}} \dot{v}(s) d s \\
& \leqslant \int_{t_{2}}^{t_{4}} z\left(s, t_{2}, r^{\prime}\right) d s<\beta\left(r^{\prime}\right)-\alpha\left(r^{\prime}\right) .
\end{aligned}
$$

The proof of Lemma 2 is complete.

LEMMA 3. Suppose that $(\mathbf{F})$ is valid and $T=\infty$. Then for arbitrarily given $r, t_{1}\left(0<r<a(\varrho), t_{1} \geqslant t_{0}\right)$, if $v(t) \leqslant r$ for $t \in\left[t_{1}, \infty\right)$ and $f\left(s, v\left(t_{1}+s\right)\right)$ $\leqslant r$ for $s \in R^{-}$, then there exists $t_{2} \in\left[t_{1}, T_{2}\left(t_{1}, r\right)\right]$ such that $v\left(t_{2}\right) \leqslant \alpha(r)$.

Proof. Assume that our lemma is not true for some $0<r<a(\varrho)$ and $t_{1} \geqslant t_{0}$. Then $\alpha(r)<v(t) \leqslant r$ for $t \in\left[t_{1}, T_{2}\left(t_{1}, r\right)\right]$. Similar reasonings as in (2.2) show that for all $t \in\left[t_{1}, T_{2}\left(t_{1}, r\right)\right]$ we have $f(s, v(t+s)) \leqslant r$ 
for $s \in R^{-}$. Hence $x_{t}\left(t_{0}, \varphi_{0}\right) \in Z\left(t, t_{1}, r\right)$ for $t \in\left[t_{1}, T_{2}\left(t_{1}, r\right)\right]$. By using

(F) we obtain:

$$
\begin{aligned}
a(r)<v\left(T_{2}\left(t_{1}, r\right)\right)=v\left(T_{1}\left(t_{1}, r\right)\right) & +\int_{T_{1}\left(l_{1}, r\right)}^{T_{2}\left(l_{1}, r\right)} v(t) d t \\
& \leqslant r+\int_{T_{1}\left(l_{1}, r\right)}^{T_{2}\left(t_{1}, r\right)} z\left(t, t_{1}, r\right) d t \leqslant r+a(r)-r
\end{aligned}
$$

which is a contradiction.

Proof of Theorem $1.1^{\circ}$ We first prove the statement concerning the stability.

For a given $\varepsilon$ with $0<\varepsilon<\varrho$ and $t_{0} \in R^{+}$put $\delta\left(\varepsilon, t_{0}\right)=b_{t_{0}}^{-1}\left(a\left(\beta^{-1}(a(\varepsilon))\right)\right)$. Here the functions $b_{t_{0}}, \alpha, \beta$ are given in the assumptions of the theorem, the function $a$ belongs to $K$ and $a(|x|) \leqslant V(t, x)$ for $t \in R^{+}, x \in R^{n}$. We prove that the number $\delta\left(\varepsilon, t_{0}\right)$ so defined satisfies the requirements formulated in the definition of stability.

Let $\varphi_{0} \in \bar{C}_{\delta\left(\varepsilon, t_{0}\right)}$ and let $x\left(t_{0}, \varphi_{0}\right)$ be a non-continuable solution on $(-\infty, T)$. Then

$$
v\left(t_{0}\right)=V\left(t_{0}, x\left(t_{0} ; t_{0}, \varphi_{0}\right)\right)=V\left(t_{0}, \varphi_{0}\right) \leqslant \alpha\left(\beta^{-1}(a(\varepsilon))\right) .
$$

From Lemma 2 with $t_{1}=t_{0}$ and $r=\beta^{-1}(a(\varepsilon))$ we get

$$
v(t)=V\left(t, x\left(t ; t_{0}, \varphi_{0}\right)\right) \leqslant a(\varepsilon), \quad t \in\left[t_{0}, T\right) .
$$

The positive defineteness of $V$ shows that from (2.4) follows $\left|x\left(t ; t_{0}, \varphi_{0}\right)\right|$ $\leqslant \varepsilon, t \in\left[t_{0}, T\right)$. The assumptions on (1.1) given in Section 1 ensure $T=\infty$; therefore the zero solution of (1.1) is stable.

$2^{\circ}$ Let $\varrho^{\prime}$ with $0<\varrho^{\prime}<\varrho$ be chosen arbitrarily. In order to prove the equi-asymptotic stability of the zero solution of (1.1) it will be sufficient to show that there exist sequences $\left\{s_{i}\right\}$ and $\left\{\tau_{i}\right\}$ such that $s_{i} \rightarrow 0$ and

$$
v(t) \leqslant s_{i}, \quad t \in\left[\tau_{i}, \infty\right),
$$

for $i=1,2, \ldots$, where $v(t)=V\left(t, x\left(t ; t_{0}, \varphi_{0}\right)\right)$ with $\varphi_{0} \in \bar{C}_{\delta\left(\ell^{\prime}, t_{0}\right)}$ chosen arbitrarily.

Such sequence $\left\{s_{i}\right\}$ and $\left\{\tau_{i}\right\}$ can be constructed as follows.

Define the sequence $\left\{s_{i}\right\}$ recursively by $s_{0}=a\left(\varrho^{\prime}\right), s_{i}=\beta\left(s_{i-1}\right)$, $i=1,2, \ldots$ Since $\beta \in K$ and since $\beta(r)<r, r>0$, it is easy to see that $s_{i} \rightarrow 0$ as $i \rightarrow \infty$.

To construct the sequence $\left\{\tau_{i}\right\}$ let us define $0<H(r)<\infty$ for $0<r$ $<a\left(\varrho^{\prime}\right)$ such that

$$
\sup \left\{f(s, v): 0 \leqslant v \leqslant a\left(\varrho^{\prime}\right)\right\} \leqslant r \quad \text { if } s \leqslant-H(r) .
$$


Let $\tau_{0}=t_{0}$ and $\tau_{i}=T_{2}\left(\tau_{i-1}+H\left(s_{i-1}\right), s_{i-1}\right), i=1,2, \ldots\left(T_{2}(t, r)\right.$ is given in assumption (F) of Theorem 1 ).

We shall prove (2.5) by induction. For $i=0$ (2.5) follows from (2.4) with $\varepsilon=\varrho^{\prime}$ and $T=\infty$. Assume (2.5) for $i=0,1, \ldots, j-1(1 \leqslant j)$; we shall prove it for $i=j$.

Let $u$ s apply Lemma 3 with $r=s_{j-1}$ and $t_{1}=\tau_{j-1}+H\left(s_{j-1}\right)$. The conditions of Lemma 3 are satisfied, since if $s \in\left(-H\left(s_{j-1}\right), 0\right]$, then

$$
f\left(s, v\left(\tau_{j-1}+H\left(s_{j-1}\right)+s\right)\right) \leqslant v\left(\tau_{j-1}+H\left(s_{j-1}\right)+s\right) \leqslant s_{j-1} .
$$

Should it happen that $s \leqslant-H\left(s_{j-1}\right)$, then according to the definition of $H\left(s_{j-1}\right)$ we would have $f\left(s, v\left(\tau_{j-1}+H\left(s_{j-1}\right)+s\right)\right) \leqslant s_{j-1}$. Hence we obtain that there exists $t_{2} \in\left[\tau_{j-1}+H\left(s_{j-1}\right), T_{2}\left(\tau_{j-1}+H\left(s_{j-1}\right), s_{j-1}\right)\right]$ such that $v\left(t_{2}\right)$ $\leqslant \alpha\left(s_{j-1}\right)$. Thus from Lemma $2\left(r=s_{j-1}, t_{1}=t_{2}\right)$ it follows that $v(t)$ $\leqslant \beta\left(s_{j-1}\right)$ for $t \in\left[t_{2}, \infty\right)$. Because of $t_{2} \leqslant T_{2}\left(\tau_{j-1}+H\left(s_{j-1}\right), s_{j-1}\right)$, we see that (2.5) holds for $i=j$ and thus, by induction, for every $i=0,1, \ldots$ Q.e.d.

By simple modifications (where necessary) of the proof of Theorem 1 we obtain:

Theorem 2. Suppose that we can find functions $V, \alpha, \beta, f, h$ with the properties formulated at the beginning of this section. Then:

$1^{\circ}$ If conditions (B), (C) hold together with

(G) there exists $b \in K$ such that

$$
V(t, x) \leqslant b(|x|), \quad t \in R, x \in R^{n},
$$

then the zero solution of (1.1) is uniformly stable.

$2^{\circ}$ If $(\mathrm{G}),(\mathrm{B}) ;(\mathrm{C}),(\mathrm{D}),(\mathrm{E}),(\mathrm{F})$ hold, then the zero solution of $(1.1)$ is strictly equi-asymptotically stable.

$3^{\circ}$ If $(\mathrm{G}),(\mathrm{B}),(\mathrm{C}),(\mathrm{D}),(\mathrm{E})$ are satisfied together with

(H) for $0<r<a(\varrho)$ there exists $T(r)>0$ and for $t \in R^{+}$there exists an interval $I(t, r) \subset[t, t+T(r)]$ such that

$$
\int_{I(t, r)} z(s, t, r) d s \leqslant a(r)-r
$$

then the zero solution of (1.1) is uniformly asymptotically stable.

$4^{\circ}$ Let (G), (B), (C), (H) be satisfied and if $\alpha(r)=\alpha r, \beta(r)=\beta r$ with some constants $\alpha, \beta, 0<\alpha<\beta<1$; suppose that there is a constant $L>0$ such that $T(r) \leqslant L$ for $0<r<a(\varrho)(T(r)$ is as in $(\mathrm{H}))$ and that there exist $B \geqslant 0, \nu>0$ such that for $0<r<a(\varrho), s \in R^{-}$, we have $\sup \{f(s, v): 0 \leqslant v$ $\leqslant r\} \leqslant B r e^{p s}$, then the zero solution of (1.1) is exponentially stable. 


\section{Some extensions and remarks}

1. In the case of equations with finite delay, the function $f$ may be neglected, because

$$
f(s, v)= \begin{cases}v, & s \in[-h, 0], \\ 0, & s \in(-\infty,-h),\end{cases}
$$

satisfies the assumptions of Theorems 1 and 2 .

2. The problem is similar in the case of some equations with infinite delay. To see this, one must allow the dependence of $f$ on $t$. Namely, if $f: R^{+} \times R^{-} \times R^{+} \rightarrow R^{+}$and if, for fixed $t, v, f(t, s, v)$ is non-decreasing in $s$, if, further, $f(t+p, s-p, v) \leqslant f(t, s, v)$ for $t, p \in R^{+}, s \in R^{-}, v \in R^{+}$, $f(t, 0, v) \leqslant v$ for $t \in R^{+}, v \in R^{+}$, and if instead of (D) the assumption

$\left(\mathrm{D}^{\prime}\right)$ for every $r>0, \bar{t} \in R^{+}$

$$
\lim _{t \rightarrow \infty}(\sup \{f(t, \bar{t}-t, v): 0 \leqslant v \leqslant r\})=0
$$

is required in Theorem 1 , then the statements on the stability properties remain valid.

Using this remark we have: If for every $t \in R^{+}$there exists $h(t) \geqslant 0$ such that $F(t, \varphi)=F(t, \psi)$ whenever $\varphi(s)=\psi(s)$ for $s \in[-h(t), 0]$, if $t-h(t) \rightarrow \infty$ as $t \rightarrow \infty$ and if the function $t-h(t)$ is non-decreasing, then

$$
f(t, s, v)= \begin{cases}v, & s \in[-h(t), 0], \\ 0, & s \in(-\infty,-h(t)),\end{cases}
$$

satisfies the assumptions of Theorem 1.

3. Using $k$ auxiliary functions $(1 \leqslant k \leqslant n)$ we can give an extension of Theorem 1. Instead of the set $Z(t, \bar{t}, r)$ we use sets with finer structure.

Let there be given functions $\omega_{1}, \omega_{2}, \ldots, \omega_{k}$ and write $\omega(t, x)$ $=\left(\omega_{1}(t, x), \ldots, \omega_{k}(t, x)\right)$. Let $\alpha, \beta \in K$ be given with $a(r)<\beta(r) \leqslant r$ for $r>0$. Further let $f: R^{-} \times R^{k} \rightarrow R^{k}$ be such that $f_{i}(s, v) \geqslant 0$ for $s \in R^{-}$, $v \in R^{k} ; f_{i}(0, v) \leqslant\left|v_{i}\right|$ for $v \in R^{k}$ and the function $f_{i}(s, v)$ is non-decreasing in $s$ for a fixed $v \in R^{k}$ for every $i=1, \ldots, k$.

Denote by $K_{r}$ the set:

$$
K_{r}=\left\{v \in R^{k}:\left|v_{i}\right| \leqslant r, i=1, \ldots, k\right\} .
$$

For a given $r>0, t \in R^{+}, \bar{t} \in R^{+}, \bar{t} \leqslant t$ and $i=1, \ldots, k$ put

$$
\begin{array}{r}
Z_{i}^{+}(t, \bar{t}, r)=\left\{\varphi \in C_{e}: \alpha(r) \leqslant \omega_{i}(t+s, \varphi(s)) \leqslant \beta(r), s \in[\bar{t}-t, 0] ;\right. \\
\omega(t+s, \varphi(s)) \in K_{r}, s \in[\bar{t}-t, 0] ; \\
\left.f(s, \omega(t+s, \varphi(s))) \in K_{r}, s \in R^{-}\right\},
\end{array}
$$




$$
\begin{array}{r}
Z_{i}^{-}(t, \bar{t}, r)=\left\{\varphi \in C_{e}:-\beta(r) \leqslant \omega_{i}(t+s, \varphi(s)) \leqslant-a(r), s \in[\bar{t}-t, 0]\right. \\
\omega(t+s, \varphi(s)) \in K_{r}, s \in[\bar{t}-t, 0] \\
\left.f(s, \omega(t+s, \varphi(s))) \in K_{r}, s \in R^{-}\right\} \\
M_{i}^{+}(t, \bar{t}, r)=\left\{\varphi \in C_{e}: a(r)<\omega_{i}(t+s, \varphi(s)) \leqslant r, s \in[\bar{t}-t, 0]\right. \\
\omega(t+s, \varphi(s)) \in K_{r}, s \in[\bar{t}-t, 0] \\
\left.f(s, \omega(t+s, \varphi(s))) \in K_{r}, s \in R^{-}\right\} \\
M_{i}^{-}(t, \bar{t}, r)=\left\{\varphi \in C_{e}:-r \leqslant \omega_{i}(t+s, \varphi(s))<-a(r), s \in[\bar{t}-t, 0]\right. \\
\omega(t+s, \varphi(s)) \in K_{r}, s \in[\bar{t}-t, 0] \\
\left.f(s, \omega(t+s, \varphi(s))) \in K_{r}, s \in R^{-}\right\}
\end{array}
$$

and

$$
\begin{aligned}
z_{i}^{+}(t, \bar{t}, r & =\sup \left\{\dot{\omega}_{i}(t, \varphi): \varphi \in Z_{i}^{+}(t, \bar{t}, r)\right\}, \\
z_{i}^{-}(t, \bar{t}, r) & =\inf \left\{\dot{\omega}_{i}(t, \varphi): \varphi \in Z_{i}^{-}(t, \bar{t}, r)\right\}, \\
m_{i}^{+}(t, \bar{t}, r) & =\sup \left\{\dot{\omega}_{i}(t, \varphi): \varphi \in M_{i}^{+}(t, \bar{t}, r)\right\}, \\
m_{i}^{-}(t, \bar{t}, r) & =\inf \left\{\dot{\omega}_{i}(t, \varphi): \varphi \in M_{i}^{-}(t, \bar{t}, r)\right\} .
\end{aligned}
$$

Further, given functions $h^{+}, h^{-}: R^{+} \rightarrow R^{+}$with $h^{+}(t), h^{-}(t) \leqslant t$, let us define

$$
\begin{aligned}
& N_{i}^{+}(t, r)=\left\{\varphi \in C_{Q}: \omega_{i}(t, \varphi(0))=\beta(r) ;\right. \\
& a(r)<\omega_{i}(t+s, \varphi(s))<\beta(r), s \in\left(h^{+}(t)-t, 0\right) ; \\
& \omega(t+s, \varphi(s)) \in K_{\beta(r)}, s \in\left(h^{+}(t)-t, 0\right) \text {; } \\
& \left.f(s, \omega(t+s, \varphi(s))) \in K_{r}, s \in R^{-}\right\} \text {, } \\
& N_{i}^{-}(t, r)=\left\{\varphi \in C_{\varrho}: \omega_{i}(t, \varphi(0))=-\beta(r) ;\right. \\
& -\beta(r)<\omega_{i}(t+s, \varphi(s))<-a(r), s \in\left(h^{-}(t)-t, 0\right) ; \\
& \omega(t+s, \varphi(s)) \in K_{\beta(r)}, s \in\left(h^{-}(t)-t, 0\right) \text {; } \\
& \left.f(s, \omega(t+s, \varphi(s))) \in K_{r}, s \in R^{-}\right\}, \\
& N_{i}(t)=\bigcup_{r>0}\left(N_{i}^{+}(t, r) \cup N_{i}^{-}(t, r)\right) .
\end{aligned}
$$

THEOREM 3. Suppose that there exist functions $\omega_{1}, \omega_{2}, \ldots, \omega_{k}, \alpha, \beta$, $f, h^{+}, h^{-}$with the above properties and that $a(|x|) \leqslant|\omega(t, x)|$ for $t \in R, x \in R^{n}$. 10 If

$\left(\mathrm{A}^{\prime}\right)$ for every $t_{0} \in R^{+}$there exists $b_{t_{0}} \in K$ such that $\left|\omega\left(t_{0}+s, \varphi(s)\right)\right|$ $\leqslant b_{t_{0}}(\|\varphi\|), \varphi \in C_{e}, s \in R^{-}$, 
(B') for every $i=1, \ldots, k$, if $t \in R^{+}, h(t)>0$ and $\varphi \in N_{i}(t)$, then $\omega_{i}(t, \varphi(0)) \dot{\omega}_{i}(t, \varphi) \leqslant 0$

$\left(\mathrm{C}^{\prime}\right)$ for every $\tau$ with $h^{+}(t) \leqslant \tau \leqslant t, h^{-}(t) \leqslant \tau \leqslant t$ resp., if the set $Z_{i}^{+}(s, \tau, r), Z_{i}^{-}(s, \tau, r)$ resp., is non-empty, $s \in[\tau, t](i=1, \ldots, k)$, then the function $z_{i}^{+}(s, \tau, r), z_{i}^{-}(s, \tau, r)$ resp., is integrable and

$$
\int_{\tau}^{t} z_{i}^{+}(s, \tau, r) d s<\beta(r)-\alpha(r), \quad \int_{\tau}^{l} z_{i}^{-}(s, \tau, r) d s>\alpha(r)-\beta(r),
$$

then the zero solution of (1.1) is stable.

$2^{\circ}$ Let $\left(\mathrm{A}^{\prime}\right),\left(\mathrm{B}^{\prime}\right),\left(\mathrm{C}^{\prime}\right)$ be satisfied, $\beta(r)<r, r>0$; suppose that for $r>0$ we have $\lim \sup \{|f(s, v)|:|v| \leqslant r\}=0$, and assume the condition:

( $\left.\mathrm{F}^{\prime}\right)$ for every $r>0, t_{0} \in R^{+}, i=1, \ldots, k$, if $M_{i}^{+}\left(t, t_{0}, r\right), M_{i}^{-}\left(t, t_{0}, r\right)$ resp., is non-empty for some $t \in\left(t_{0}, \infty\right)$, then there exist intervals $\left[T_{1, i}^{+}\left(t_{0}, r\right)\right.$, $\left.T_{2, i}^{+}\left(t_{0}, r\right)\right],\left[T_{1, i}^{-}\left(t_{0}, r\right), T_{2, i}^{-}\left(t_{0}, r\right)\right] \subset\left(t_{0}, \infty\right)$ such that $m_{i}^{+}\left(s, t_{0}, r\right), m_{i}^{-}\left(s, t_{0}, r\right)$ resp., is integrable and

$$
\int_{T_{1, i}^{+}\left(t_{0}, r\right)}^{T_{2, i}^{+}\left(t_{0}, r\right)} m_{i}^{+}\left(s, t_{0}, r\right) d s \leqslant \alpha(r)-r, \quad \int_{T_{1, i}^{-}\left(l_{0}, r\right)}^{T_{2, i}^{-}\left(l_{0}, r\right)} m_{i}^{-}\left(s, t_{0}, r\right) d s \geqslant r-\alpha(r) .
$$

Then the zero solution of (1.1) is equi-asymptotically stable.

4. Here we give another extension of Theorem 1 . In this extension $(x, y)$ denotes the scalar product of vectors $x, y \in R^{k}$ and $|x|=\sqrt{(x, x)}$. Let there be given $\omega(t, x)=\left(\omega_{1}(t, x), \ldots, \omega_{k}(t, x)\right)$ and assume that $|\omega(t, x)|$ is positive definite. Put $\alpha, \beta \in K, \alpha(r)<\beta(r) \leqslant r$ for $r>0$. Further, let $f: R^{-} \times R^{k} \rightarrow R^{+}$be such that $f(s, v) \leqslant|v|$ and $f(s, v)$ is nondecreasing for a fived $v$.

Define the set

$$
\begin{aligned}
Z(t, \bar{t}, r)=\left\{\varphi \in C_{\varrho}: \alpha(r) \leqslant\right. & |\omega(t+s, \varphi(s))| \leqslant r, \\
& \left.s \in[\bar{t}-t, 0] ; f(s, \omega(t+s, \varphi(s))) \leqslant r, s \in R^{-}\right\}
\end{aligned}
$$

and put

$$
\begin{gathered}
k(t, \bar{t}, r)=\sup \{|\dot{\omega}(t, \varphi)| /|\omega(t, \varphi(0))|: \varphi \in Z(t, \bar{t}, r)\} \\
z(t, \bar{t}, r)=\sup \{(\omega(t, \varphi(0)), \dot{\omega}(t, \varphi)) /|\omega(t, \varphi(0))|: \varphi \in Z(t, \bar{t}, r)\} .
\end{gathered}
$$

Further, write

$$
\begin{aligned}
M(t, \bar{t}, r) & =\{\varphi \in C: \varphi \in z(t, \bar{t}, r) \\
& \left.\left|\frac{\omega(t, \varphi(0))}{|\omega(t, \varphi(0))|}-\frac{\omega(t+s, \varphi(s))}{|\omega(t+s, \varphi(s))|}\right| \leqslant \int_{t+s}^{t} k(z, \bar{t}, r) d z, s \in[\bar{t}-t, 0]\right\}
\end{aligned}
$$

7 - Annales Polonicl Mathematici XXXV1.3 
and

$$
m(t, \bar{t}, r)=\sup \{(\omega(t, \varphi(0)), \dot{\omega}(t, \varphi)) /|\omega(t, \varphi(0))|: \varphi \in M(t, \bar{t}, r)\} .
$$

THEOREM 4. Assume that there exist functions $\omega, \alpha, \beta, f$ with the above properties and a function $h: R^{+} \rightarrow R^{+}$such that $h(t) \leqslant t$.

$1^{\circ}$ If $\left(A^{\prime}\right)$ holds together with:

$\left(\mathrm{B}^{\prime \prime}\right)(\dot{\omega}(t, \varphi), \omega(t, \varphi(0))) \leqslant 0$ for $t \in R^{+}$with $h(t)>0$ and for

$$
\varphi \in \bigcup_{r>0}(M(t, h(t), r) \cap\{\varphi \in C: \beta(r)=|\omega(t, \varphi(0))|\}),
$$

$\left(\mathrm{C}^{\prime \prime}\right) \int_{\tau}^{t} z(s, \tau, r) d s<\beta(r)-\alpha(r)$ for $t \in R^{+}$and $\tau \in(h(t), t]$,

then the zero solution of (1.1) is stable.

$2^{\circ}$ Let $\left(\mathrm{A}^{\prime}\right),\left(\mathrm{B}^{\prime \prime}\right)$ and $\left(\mathrm{C}^{\prime \prime}\right)$ be satisfied and $\beta(r)<r, r>0$; suppose that for $r>0$ we have $\limsup \{f(s, v):|v| \leqslant r\}=0$, and assume the condition:

$\left(\mathbf{F}^{\prime \prime}\right)$ for every $r>0, t_{0} \in R^{+}$there exists an interval $\left[T_{1}\left(t_{0}, r\right), T_{2}\left(t_{0}, r\right)\right]$ $\subset\left(t_{0}, \infty\right)$ such that

$$
\int_{T_{1}\left(i_{0}, r\right)}^{T_{2}\left(l_{0}, r\right)} m(s, \tau, r) d s \leqslant \alpha(r)-r .
$$

Then the zero solution of (1.1) is equi-asymptotically stable.

5. Our results can also be reformulated so as to involve partial stability.

Let $P: R^{n} \rightarrow R^{n}$ be a projection. The zero solution of (1.1) will be called $P$-stable at $t_{0}$, if instead of $(1.2)$ it satisfied the condition $\left|P x\left(t ; t_{0}, \varphi_{0}\right)\right|$ $\leqslant \varepsilon$ for $t \in\left[t_{0}, \infty\right)$. Analogously there can be defined the properties of $\boldsymbol{P}$-stability, equi-asymptotical $\boldsymbol{P}$-stability etc.

It can be easily seen that if in our theorems instead of the positive definiteness of $|\omega(t, x)|$ we require $a(|P x|) \leqslant|\omega(t, x)|$ for $t \in R, x \in R^{n}$, where $a \in K$, then we can state the corresponding $\boldsymbol{P}$-stability property.

\section{Some applications}

In this section we give some applications of our theorems without proofs; we construct only the auxiliary functions, where it seems to be necessary.

CoRollary 1. If there exist a function $V$ and, for every $t_{0} \in R^{+}$, a function $b_{i_{0}} \in K$ such that $V\left(t_{0}+s, \varphi(s)\right) \leqslant b_{i_{0}}(\|\varphi\|)$ for $s \in R^{-}, \varphi \in C$ and $\dot{V}(t, \varphi) \leqslant 0$ on the set

$$
\left\{(t, \varphi): V(t+s, \varphi(s)) \leqslant V(t, \varphi(0)), s \in R^{-}\right\}
$$


then the zero solution of (1.1) is stable. If there exists $b \in K$ such that $\nabla(t, x)$ $\leqslant b(|x|)$ for $t \in R, x \in R^{n}$, then the zero solution of (1.1) is uniformly stable.

This result is an extension of Razimikhin's and Seifert's result mentioned in Section 1.

Set $\beta(r)=r, \alpha(r)<r, h(t)=t$ and $f(s, v)=v$. With these functions the stability of the zero solution follows from Theorem 1 and the uniform stability follows from Theorem 2 .

Corollary 2. Suppose that there exist a function $V$, functions $b, c \in K$,

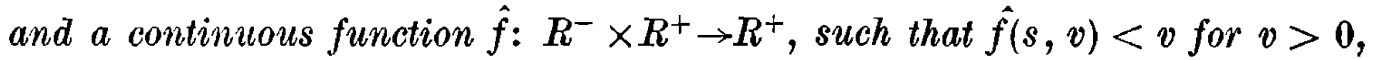
$\lim \sup \{\hat{f}(s, v): v \leqslant r\}=0, \hat{f}(s, v)$ is non-decreasing in $s$ for a fixed $v$, $\hat{f}(0, v)$ is non-decreasing, $V(t, x) \leqslant b(|x|)$ for $t \in R, x \in R^{n}$, and $\dot{V}(t, \varphi)$ $\leqslant-c(|\varphi(0)|)$ on the set

$$
\left\{(t, \varphi): \hat{f}(s, V(t+s, \varphi(s)))<V(t, \varphi(0)), s \in R^{-}\right\} .
$$

Then the zero solution of (1.1) is uniformly asymptotically stable.

In particular, if (1.1) is an equation of finite delay and if

$$
\hat{f}(s, v)= \begin{cases}\bar{f}(v), & s \in[-h, 0] \\ 0, & s \in(-\infty,-h),\end{cases}
$$

where $0 \leqslant \bar{f}(v)<v, \bar{f}(v)$ is non-decreasing, then the above corollary implies then theorem of Krasovskil and Driver mentioned in the introduction.

Put $\alpha(r)=\hat{f}(0, r)$. Define a function $g: R^{-} \times R^{+} \rightarrow R^{+}$by $\hat{f(s, v)}$ $=\hat{f}(0, g(s, v))$ and let $f(s, v)=\min (g(s, v), v)$. Choose $\beta \in K$ such that $\alpha(r)<\beta(r)<r$ for $r>0$. Finally, set $h(t)=t, t \in R^{+}$. Then this corollary follows from Theorem 2 .

COROLlaRY 3. Consider the scalar equation

$$
\dot{x}(t)=p(t) x(t)+q(t) x(t-\tau(t)),
$$

where functions $p, q, \tau$ are continuous on $R^{+}$. If $p(t) \leqslant 0, q(t) \leqslant 0,0 \leqslant \tau(t)$ $\leqslant h$ ( $h$ constant) for $t \in R^{+}$, and if there exists $\alpha, 0<\alpha<1$, with

$$
\sup \left\{\int_{t=\tau}^{t}(\alpha p(s)+|q(s)|) d s: t \in R^{+}, \tau \in[0, \min (\tau(t), t)]\right\}<1-\alpha
$$

then the zero solution of (1.1) is uniformly stable. If, moreover, $\int_{0}^{\infty}(p(s)+$ $+q(s)) d s=-\infty$, then the zero solution is strictly equi-asymptotically stable. 
Equation (4.1) was investigated by Mikołajska [7], who proved that if

$$
\int_{t-\tau}^{\infty}(\alpha p(s)+|q(s)|) d s<1-\alpha, \quad t \in R^{+}, \tau \in[0, \min (\tau(t), t)],
$$

then the zero solution is stable at 0 .

Put $V(t, x)=|x|, \alpha(r)=\alpha \cdot r, \beta(r)=\beta r$, where $0<\alpha<\beta<1$ are constants. $f$ is given as in Remark $1, h(t)=(t-\tau(t))^{+}$. Then Theorem 2 can be applied. (The assumptions required in Section 1 are satisfied by (4.1); see e.g. Hale [3], Theorem 3.1 and Theorem 4.2.)

COROLLARY 4. Consider equation (4.1). If $q(t) \leqslant 0,0 \leqslant \tau(t) \leqslant h$, $\int_{(\ell-\tau(l))^{+}}^{t}|q(s)| d s \leqslant C<1$ for $t \in R^{+}$

$$
\int_{0}^{\infty} p^{+}(s) d s<\infty \quad \text { and } \quad \int_{0}^{\infty}(p(s)+q(s)) d s=-\infty,
$$

then the zero solution is strictly equi-asymptotically stable. Without the last assumption the zero solution is uniformly stable.

It was proved by Mikołajska [7] without the last assumption that the zero solution is stable at 0 .

The statement follows from Theorem 2 with

$$
\begin{aligned}
V(t, x) & =|x| \exp \left(-\int_{0}^{t} p^{+}(s) d s\right), & \alpha(r) & =\frac{1-C}{4} r, \\
\beta(r) & =\left(C+\frac{1-C}{2}\right) r, & h(t) & =(t-\tau(t))^{+} .
\end{aligned}
$$

In the above corollaries, instead of the boundedness of $\tau(t)$, it is sufficient to require $t-\tau(t) \rightarrow \infty$ as $t \rightarrow \infty$.

COROLLARY 5. Consider the scalar equation

$$
\dot{x}(t)=f(t, x(t-\tau(t)))+g\left(t, x_{t}\right),
$$

where $f: R^{+} \times R \rightarrow R, g: R^{+} \times C \rightarrow R$. Assume $0 \leqslant \tau(t) \leqslant h$ for $t \in R^{+}$ $(h=$ constant $)$,

$$
\begin{gathered}
x f(t, x) \leqslant-a(t) x^{2}, \quad t \in R^{+}, x \in R, \\
|g(t, \varphi)| \leqslant \theta a(t) \sup \left\{|\sigma(s) \varphi(s)|: s \in R^{-}\right\}, \quad t \in R^{+}, \varphi \in C,
\end{gathered}
$$

where $\theta$ is a constant, $0<\theta<1,0 \leqslant \sigma(s) \leqslant 1$ for $s \in R^{-}, \sigma(s) \rightarrow 0$ as $s \rightarrow-\infty$,

$$
\int_{(t-\tau(t))^{+}}^{t} a(s) d s \leqslant c<\frac{1-\theta}{1+\theta}, \quad t \in R^{+} \quad(c=\text { constant })
$$

and $\int_{0}^{\infty} a(s) d s=\infty$. Then the zero solution is equi-asymptotically stable. 
In particular, if $\tau(t)=0$ and if

$$
|g(t, \varphi)| \leqslant \theta a(t) \sup \{|\varphi(s)|: s \in[-h, 0]\} \quad \text { for } t \in R^{+}, \varphi \in C,
$$

then (4.2) and the condition $\int_{0}^{\infty} a(s) d s=\infty$ imply the equi-asymptotic stability. Winston [10] investigated the above equation using the latter assumptions (but (4.2) was in a sharper from) and proved the asymptotic stability.

Let $N$ be so large that

$$
\frac{N-2}{N} \frac{1-\theta}{1+\theta}>c
$$

and define

$$
\begin{aligned}
& \alpha(r)=\left(\theta+\frac{1-\theta}{2}\right) r, \quad \beta(r)=\left(\theta+\frac{N-1}{N}(1-\theta)\right) r, \\
& V(t, x)=|x|, \quad f(s, v)=\sigma(s) v, \quad h(t)=(t-\tau(t))^{+} .
\end{aligned}
$$

The statement follows from Theorem 2.

CoROLlaRY 6. Consider the equation $\dot{x}(t)=A x(t)+B x(t-\tau)$, where $\tau>0$ is a constant. $A$ and $B$ are $n$ by $n$ matrices.

As regards this equation, Hale [3] obtained a result concerning the asymptotic stability, in the case where the eigenvalues of $A$ have negative real parts. Here we present a result assuming that the real parts of the eigenvalues of $B$ are negative.

Let $D$ be a solution of $D^{2} B+{ }^{T} B D^{2}=-2 E$ ( $E$ is the unit matrix, $D$ exists and is positive definite [5]). Denote the greatest eigenvalue of $D$ by $\lambda$, and that of $D^{-1} \frac{D^{2} A+{ }^{T} A D^{2}}{2} D^{-1}$ by $\mu$.

If there exists an $\alpha, 0<\alpha<1$, such that

$$
\begin{aligned}
&\left|D A D^{-1}\right|+\left|D B D^{-1}\right|<(1-\alpha) / \tau \quad \text { and } \\
& \mu^{+}+\tau\left(\left|D A D^{-1}\right|+\left|D B D^{-1}\right|\right) / \lambda<\alpha\left(\mu^{-}+1 / \lambda^{2}\right),
\end{aligned}
$$

then the zero solution of the above equation is equi-asymptotically (even exponentially) stable.

This assertion follows from Theorem 4 by

$$
\begin{aligned}
\omega(t, x)=D x, \quad h(t) & =[t-\tau]^{+}, \quad \alpha(r)=a \cdot r, \quad \beta(r)=\beta \cdot r, \\
f(s, v) & = \begin{cases}v, & s \in[-\tau, 0], \\
0, & s \in(-\infty,-\tau),\end{cases}
\end{aligned}
$$

where $\beta$ is a constant such that $0<a<\beta<1$ and $\left|D A D^{-1}\right|+\left|D B D^{-1}\right|$ $<(\beta-\alpha) / \tau$. 
For example, if $A=0, B$ is synmetrical and

$$
\tau|B|<\frac{1+2 \lambda-\sqrt{1+4 \lambda}}{2 \lambda},
$$

where $-\lambda$ is the greatest eigenvalue of $B$, then the zero solution is exponentially stable.

We remark that in the case of $A=0, B$ symmetrical (the eigenvalues of $B$ are negative), then, using Theorem 3 , we can prove the asymptotic stability in the case $\tau|B|<1$. If the eigenvalues of $B$ are not all negative, but the zero solution of $\dot{x}(t)=B x(t)$ is asymptotically $P$-stable $(\tau|B|<1)$, then also the trivial solution of $\dot{x}(t)=B x(t-\tau)$ is asymptotically $P$-stable.

\section{References}

[1] R. Driver, 1 functional-differential equation arising in a two body problem of electrodynamics, International Symposium of Nonlinear Differential Equations and Nonlinear Mechanics, Academic Press, 1963, p. 474-484.

[2] A. Halanay and J. A. Yorke, Some new results and problems in the theory of differential-delay equations, SIAM Rieview 13 (1971), p. 58-80.

[3] J. K. II ale, Functional differential equations, Springer-Verlag, New York 1971.

[4] Н. Н. І р асовский, Некоторые задачи теории устойчивости двизссния, физматгиз, Москва 1959.

[5] А. М. ЈІ я пу нов, Общал зидача об устойчивости дөижениия, Г'остехиздит, Москва 1950.

[6] Z. Mikolajska, Une remarque sur des notes de Razumichin et Kraso'skij sur la stabilité asymptotique, Ann. Polon. Math. 22 (1969), p. 69-72.

[7] - Une modification de la condition de Liapounov pour les équations à paramètre retardé, ibidem 21 (1969), p. 103-111.

[8] Б. С. Разимихин, Об устойчивости систем с запаздьванием, ІІрикл. матх. мех. 20 (1959). p. 500-512.

[9] G. Seifert, Liapunov-Razumilchin conditions for stability and boundedness of functional differential equations of Vollerra type, J. of Diff. Eq. 14 (1973), p. 424430 .

[10] E. Winston, Asymptotic stability for ordinary differential equations with delayed perturbations, SIAM J. Math. Anal. 5 (1974), p. 303-308.

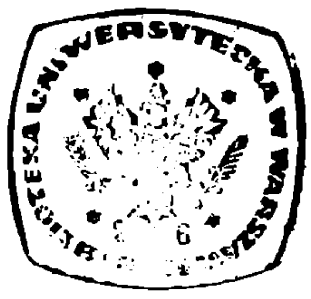

